\title{
Modelagem da espessura e percentual de casca em árvores de Tectona grandis L.f
}

\author{
Modeling the thickness and percentage of bark on trees \\ of Tectona grandis L.f
}

\author{
Diogo Guido Streck Vendruscolo ${ }^{1}$, Ronaldo Drescher ${ }^{1}$, \\ Samuel de Pádua Chaves e Carvalho ${ }^{1}$, Reginaldo Antonio Medeiros ${ }^{2}$ \\ e Rômulo Môra ${ }^{1}$
}

\begin{abstract}
Resumo
Objetivou-se nesse trabalho avaliar modelos e variáveis para a modelagem da espessura e conteúdo percentual de casca em árvores de Tectona grandis (teca). Os dados foram coletados de 68 árvores dominantes de teca com idade variando de 6 a 34 anos, distribuídas em diferentes regiões de Mato Grosso. Para estimar a espessura da casca das árvores foram testados dois modelos de regressão, sendo um polinomial e outro linear simples. Em ambos os modelos também foi inserida a idade como um componente aleatório. O percentual de casca foi estimado utilizando modelos da família exponencial. A qualidade dos ajustes foi realizada por meio de parâmetros estatísticos e um conjunto de analises gráficas. Verificou-se que o modelo polinomial com a variável independente altura relativa e a idade como efeito aleatório mostrou-se eficiente para a predição da espessura da casca ao longo do fuste de árvores de teca. O conteúdo percentual de casca das árvores em pé pode ser estimado com precisão, por meio de um modelo biexponencial utilizando apenas o DAP como variável regressora.
\end{abstract}

Palavras-chave: Teca; árvores dominantes; volume de casca; modelagem de efeitos mistos; modelos não lineares.

\begin{abstract}
The objective of this work was to evaluate models and variables for modeling the thickness and percentage content of bark in Tectona grandis (teak) trees. The data were collected from 68 dominant teak trees with ages varying from 6 to 34 years, distributed in different regions of Mato Grosso. To estimate of trees bark thickness, two models were tested using a polynomial regression model and a simple linear regression model. In both models, age was also inserted as a random component. The percentage of bark was estimated using exponential family models. The quality of the adjustments was made through statistical criteria and a set of graphical analyzes. It was verified that the polynomial model with the independent variable relative height and age as random effects proved to be efficient for the prediction of the thickness of the bark along the stem of the teak trees. The percentage bark content of standing trees can be accurately estimated by means of a bi-exponential model using only the $D B H$ as the regressor variable.
\end{abstract}

Keywords: Teak, dominant trees, bark volume, mixed effects modeling, nonlinear models.

\section{INTRODUÇÃO}

A teca (Tectona grandis) é amplamente utilizada em reflorestamento nas regiões tropicais da América, Ásia e África. Estima-se uma área plantada com a espécie é de aproximadamente 4,35 milhões de hectares distribuídos em 52 países tropicais diferentes (FAO, 2015). A madeira da teca bem conhecida por sua característica mecânica, resistência ao ataque de xilófagos e aspectos estéticos, o que torna a essa cultura uma atração por parte de grandes investidores do setor privado (MOYA; BOND; QUESADA-PINEDA, 2014).

Grande parte da madeira da teca vem de plantações clonais de rápido crescimento e manejadas com rotações mais curtas $(\mathrm{FAO}, 2015)$. Plantios florestais de crescimento rápido exigem um planejamento

1. Departamento de Engenharia Florestal, Universidade Federal de Mato Grosso - UFMT. Cuiabá / MT, Brasil. e-mail: diogoguido@hotmail.com

2. Departamento de Engenharia Florestal. IFMT - Instituto Federal de Mato Grosso, Campus Cáceres. Cáceres / MT, Brasil.

Sci. For., Piracicaba, v. 47, n. 121, p. 139-149, mar. 2019 DOI: dx.doi.org/10.18671/scifor.v47n121.14 
Vendruscolo et al. - Modelagem da espessura e percentual de casca em

árvores de Tectona grandis L.f

silvicultural intensivo e um acompanhamento rigoroso do crescimento e da produção por meio de inventários florestais contínuos.

Durante os inventários florestais os diâmetros das árvores geralmente são medidos com casca, no entanto, muitos mercados exigem a quantificação do volume de madeira sem casca (STÄNGLE; SAUTER; DORMANN et al., 2017). Tradicionalmente, as estimativas do volume de casca das árvores são consideradas de importância secundária, tendo em vista que na maioria dos casos esta é descartada para efeito de transformação industrial da madeira. Porém graças a inversão do conceito de que a casca era um resíduo indesejado para recentemente ser considerada uma alternativa de combustível ou fonte de biomateriais de alto valor, há interesse em quantificar esse produto já que este pode fornecer rendimentos adicionais para a indústria de base florestal (DORUSKA et al., 2009; JANG et al., 2016).

Estudos apontam que a rejeição do volume de casca ou estimação inadequada da mesma pode acarretar em erros multiplicativos gerando impacto significativo sobre o volume das árvores, pois para algumas espécies a casca pode compreender um grande percentual do volume do tronco (HARKIN; ROME, 1971; LI; WEISKITTEL, 2011). No caso da teca o percentual de casca chega a representar valores próximos a $30 \%$ do volume total das árvores em idades iniciais ou em árvores de menor diâmetro (LEITE et al., 2011).

A espessura de casca pode ser estimada por equações, em função do diâmetro com casca, altura relativa no tronco, diâmetro a 1,3 m do solo e altura total da árvore (KITIKIDOU et al., 2014; LI; WEISKITTEL, 2011). Assim, conhecendo o volume com casca, pode-se aplicar modelos de espessura da casca, para finalmente obter os volumes sem casca. Portanto, modelos que forneçam estimativas precisas da quantidade de casca de árvores em pé são muito úteis em estudos de crescimento e produção (KITIKIDOU et al., 2014).

Pesquisas realizadas para estimar a espessura de casca em teca utilizam equações em função do diâmetro da árvore (CORDERO; KANNINEN, 2003; TEWARI; MARISWAMY, 2013). No entanto, trabalhos com outras espécies mostram que a espessura da casca varia de acordo com a idade e da base para o topo da árvore (BROOKS; JIANG, 2009; LAASASENAHO et al., 2005). Assim, a utilização de modelos que utilizem estas variáveis pode fornecer predições de espessura de casca mais acuradas.

Neste estudo, objetivou-se testar diferentes modelos e variáveis para modelar a espessura e o conteúdo percentual de casca em árvores de Tectona grandis.

\section{MATERIAL E MÉTODOS}

Este trabalho foi realizado com dados oriundos de plantios comerciais e experimentais com teca de origem seminal, distribuídos em diferentes localidades no estado Mato Grosso (Figura 1). As regiões onde os povoamentos estão implantados apresentam clima tropical Aw segundo a classificação de Köppen, com inverno seco, verão chuvoso e temperatura média anual de variando de 24 a $26{ }^{\circ} \mathrm{C}$, A precipitação total anual varia em média de 1.900 a 2.200 no povoamento localizado mais ao norte e de 1.600 a 1.900 nos demais. Os maiores índices de chuva no estado de Mato Grosso ocorrem entre os meses de dezembro a março (ALVARES et al., 2013).

Os dados foram coletados de 68 árvores dominantes de teca com idades de 6, 7, 10, 16, 17 e 34 anos. O número de árvores amostradas em cada idade foram de 10,15, 9, 10, 16 e 8 respectivamente. As medidas de espessura de casca (EC) foram obtidas de discos utilizados em análise de tronco em outras pesquisas (CHAVES et al., 2016; DRESCHER et al., 2016; GAVA, 2015; VENDRUSCOLO, 2017). Em todas as ocasiões, os discos foram retirados a partir de uma altura mínima de 0,1 m; 1,3 m; até um diâmetro com casca de aproximadamente $5 \mathrm{~cm}$ e distâncias intervalares de no máximo $3 \mathrm{~m}$. Assim, a base de dados foi composta pela medição da casca em quatro posições perpendiculares e posteriormente calculada a média aritmética dessas medidas em cada disco. Também foram coletadas as informações de diâmetro a 1,3 m do solo (DAP) e altura total de cada árvore. A Tabela 1 fornece um resumo estatístico do conjunto de dados.

Para estimar a espessura da casca das árvores foram testados dois modelos de regressão, sendo um polinomial (Modelo 1) e outro linear simples (Modelo 2). A variável dependente para os dois modelos foram à espessura de casca $(E C \mathrm{em} \mathrm{cm})$, enquanto a independente para o polinômio foi à altura relativa (variando de 0 a 1) e para o linear simples foi o diâmetro (di). 
Tabela 1. Estatísticas resumidas das árvores amostradas para a modelagem da casca.

Table 1. Summary statistics of the trees sampled for bark modeling.

\begin{tabular}{ccccc}
\hline Variável & Média & Desv. Padrão & Mínimo & Máximo \\
\hline Idade (anos) & 15,00 & 9,62 & 6 & 34 \\
Altura $(m)$ & 18,11 & 4,85 & 10,90 & 28,80 \\
$d i_{c c}-D$ & 14,26 & 8,11 & 2,35 & 57,75 \\
$d i_{s c}-d$ & 13,31 & 7,79 & 1,99 & 55,55 \\
$D A P_{c c}$ & 21,35 & 8,92 & 10,10 & 51,06 \\
$D A P_{s c}$ & 20,40 & 8,77 & 8,74 & 50,90 \\
$E C=(D-d) / 2$ & 0,67 & 0,18 & 0,39 & 1,08 \\
\hline
\end{tabular}

$\mathrm{di}_{\mathrm{cc}}=$ diâmetro com casca $(\mathrm{cm}) ; \mathrm{di}_{\mathrm{sc}}=$ diâmetro sem casca $(\mathrm{cm}) ; D A P_{\mathrm{cc}}=$ diâmetro a I,3 $\mathrm{m}$ de altura com casca $(\mathrm{cm}) ;$ DAP ${ }_{\mathrm{sc}}=$ diâmetro a I,3 m de altura sem casca $(\mathrm{cm}) ; E C=$ espessura da casca $(\mathrm{cm})$.

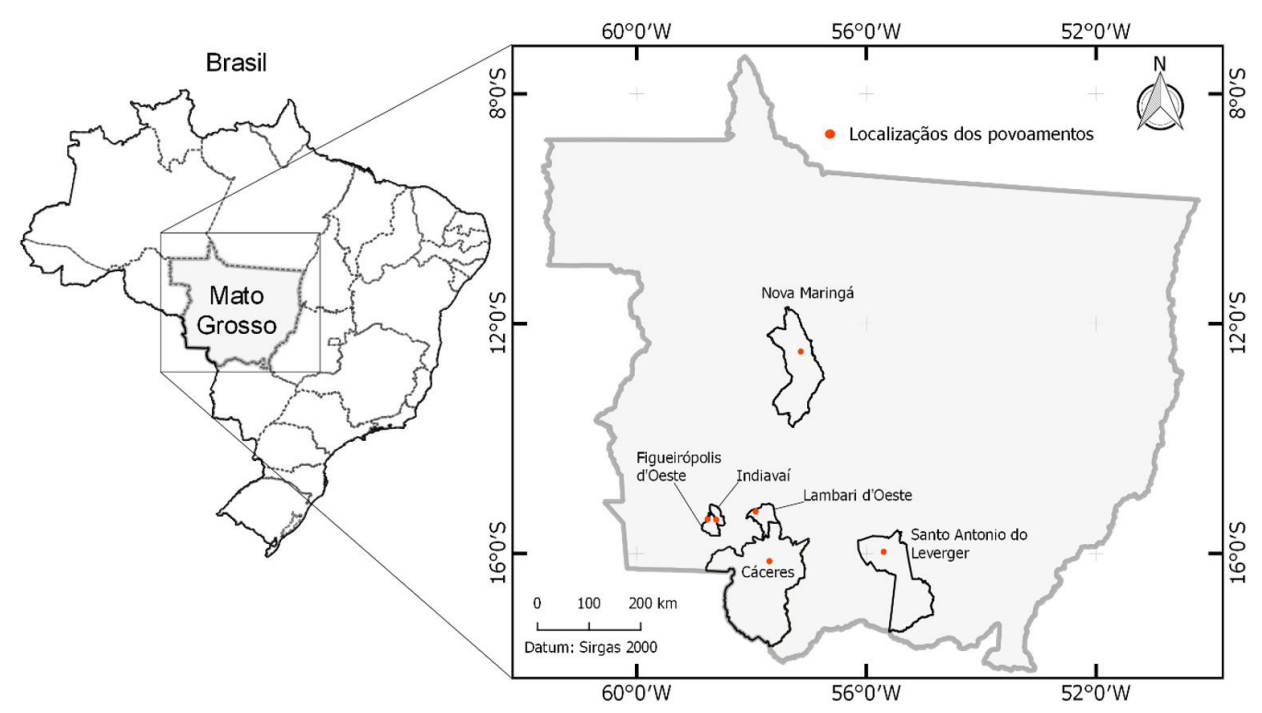

Figura 1. Distribuição dos povoamentos amostrados no estado de Mato Grosso, Brasil.

Figure 1. Distribution of sampled stands in the state of Mato Grosso, Brazil.

$$
\begin{gathered}
E C_{i j}=\beta_{0}+\beta_{1} h_{i j}+\beta_{2} h_{i j}^{2}+\beta_{3} h_{i j}^{3}+\beta_{4} h_{i j}^{4}+\beta_{5} h_{i j}^{5}+\varepsilon_{i j} \\
E C_{i j}=\beta_{0}+\beta_{I} d i_{i j}+\varepsilon_{i}
\end{gathered}
$$

em que: $E C_{i j}=$ espessura da casca da $i$-ésima árvore na $j$-ésima posição do fuste $(\mathrm{cm}) ; \beta_{i s}=$ parâmetros a serem estimados por regressão; $h_{i j}=$ altura relativa da $i$-ésima árvore na $j$-ésima posição do fuste; $d i_{i j}=$ diâmetro da $i$-ésima árvore na $j$-ésima posição do fuste e $\varepsilon_{i}=$ erro aleatório.

Conforme preconizado por Laasasenaho et al. (2005), a espessura da casca é correlacionada diretamente com a idade das árvores. Dessa forma, foi inserido nos procedimentos de ajuste, o efeito da idade como um componente aleatório do modelo fixo (Modelo 1 e 2), resultando nos Modelos 3 e 4 .

$$
\begin{gathered}
E C_{i j}=\left(\beta_{0}+b_{0 i}\right)+\left(\beta_{1}+b_{I i}\right) h_{i j}+\left(\beta_{2}+b_{2 i}\right) h_{i j}^{2}+\left(\beta_{3}+b_{3 i}\right) h_{i j}^{3}+\left(\beta_{4}+b_{4 i}\right) h_{i j}^{4}+\left(\beta_{5}+b_{5 i}\right) h_{i j}^{5}+\varepsilon_{i j} \\
E C_{i j}=\left(\beta_{0}+b_{0 i}\right)+\left(\beta_{I}+b_{I i}\right) d i_{i j}+\varepsilon_{i}
\end{gathered}
$$

em que: $\beta$ = vetor de efeitos fixos que correspondem as alturas relativas das árvores e/ou diâmetros; $b=$ vetor de efeitos aleatórios que correspondem as diferentes idades da i-ésima árvore; e $\varepsilon_{i}=$ erro aleatório entre os grupos.

Conforme descrito por Pinheiro e Bates (2000), " $i$ " varia de 1 até M (valor associado a cada variável em cada grupo), $b i \sim \mathrm{N}(0, \Sigma)$ e $\varepsilon i \sim \mathrm{N}\left(0, \sigma^{2} I\right)$.

Posteriormente, por meio da fórmula de Smalian foram calculados os volumes com e sem casca das árvores, visando ajustar e testar dois modelos de regressão, sendo um exponencial (5) e outro biexponencial (6) que possibilitem gerar estimativas de porcentagem de casca das árvores em pé utilizando apenas o diâmetro a 1,3 m de altura (DAP) como variável preditora. 
Vendruscolo et al. - Modelagem da espessura e percentual de casca em

árvores de Tectona grandis L.f

$$
\begin{gathered}
\%_{C A S C A_{i}}=\beta_{0} \exp \left(-\exp \left(\beta_{1}\right) D A P_{i}\right)+\varepsilon_{i} \\
\%_{C A S C A_{i}}=\beta_{0} \exp \left(-\exp \left(\beta_{1}\right) D A P_{i}\right)+\beta_{2} \exp \left(-\exp \left(\beta_{3}\right) D A P_{i}\right)+\varepsilon_{i}
\end{gathered}
$$

em que: $\%_{C A S C A i}=$ porcentagem de casca da $i$-ésima árvore; $\beta_{i}=$ coeficientes do modelo; exp = exponencial; $D A P_{i}=$ diâmetro a $1,3 \mathrm{~m}$ de altura $(\mathrm{cm})$ da $i$-ésima árvore e $\varepsilon_{i}=$ erro aleatório.

As estimativas dos parâmetros de regressão dos modelos lineares foram obtidas pelo método dos mínimos quadrados, enquanto, para os modelos não lineares empregou-se o método iterativo utilizando o algoritmo de Gauss-Newton. A acurácia das estimativas obtidas pelas equações foi avaliada de acordo com as seguintes estatísticas: coeficiente de correlação $\left(r_{\hat{y y y}}\right)$, erro padrão relativo $($ RQEM\%), significância dos coeficientes de regressão $(\beta)$ e a análise gráfica dos resíduos, calculados pelas Equações 7, 8 e 9 respectivamente:

$$
\begin{gathered}
r_{y \hat{y}}=\frac{\operatorname{cov}(y, \hat{y})}{\sqrt{S^{2}(y) S^{2}(\hat{y})}} \\
\operatorname{RQEM\% }=\frac{100}{\bar{y}} \sqrt{\frac{\sum_{i=l}^{n}\left(y_{i}-\hat{y}_{i}\right)^{2}}{n}} \\
\text { Residuo } \%=\left(\frac{y_{i}-\hat{y}_{i}}{y_{i}}\right) 100
\end{gathered}
$$

em que: $\operatorname{cov}=$ covariância; $y_{i}=$ variável dependente observada; $\hat{y}_{i}=$ variável dependente estimada; $S^{2}=$ variância; $\bar{y}=$ média da variável dependente observada; $n=$ número de observações.

Complementarmente, para auxiliar na escolha da melhor equação, além das análises gráficas residuais, também foram avaliados os gráficos dos valores observados versus estimados e histograma de frequência dos erros relativos. Nas análises estatísticas utilizou-se um nível de $5 \%$ de significância, sendo todos os procedimentos desenvolvidos em linguagem R de programação (R CORE TEAM, 2017).

\section{RESULTADOS E DISCUSSÃO}

Na Figura 2 estão representados os pontos referentes à espessura da casca (EC) em função da altura relativa das árvores para as diferentes idades e na Figura 3 a espessura da casca em função do diâmetro $(d i)$.

A relação observada entre a EC e a altura relativa das árvores induz a uma semelhança com o afilamento do fuste, o que explica a adequabilidade do modelo polinomial para descrever tal relação funcional.

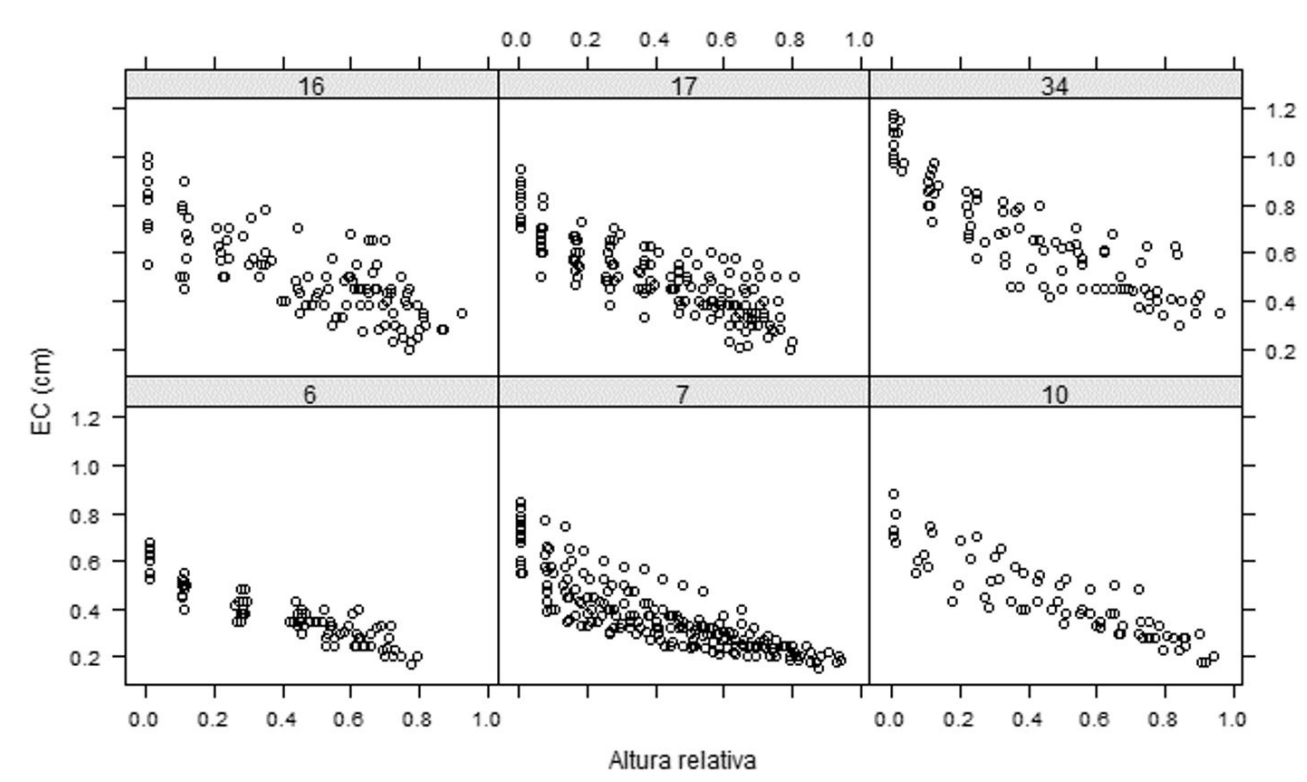

Figura 2. Relação entre a espessura da casca $(E C)$ e a altura relativa das árvores de Tectona grandis em diferentes idades.

Figure 2. Relationship between bark thickness $(E C)$ and relative height of Tectona grandis trees at different ages. 


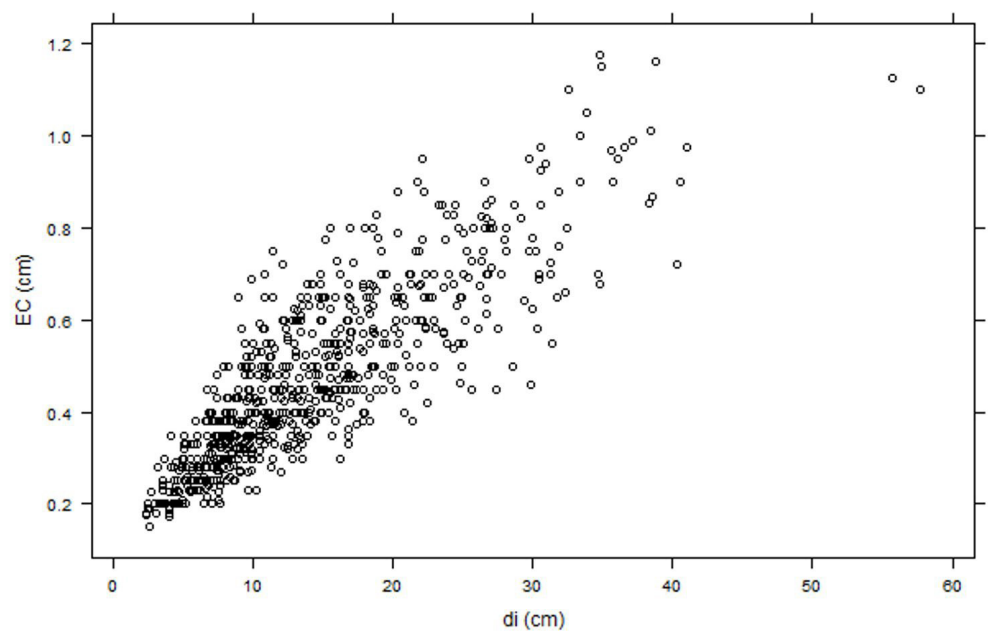

Figura 3. Relação entre a espessura da casca $(E C)$ e o diâmetro $\left(d_{i}\right)$ das árvores de Tectona grandis.

Figure 3. Relationship between the thickness of the bark $(E C)$ and the diameter $(d i)$ of Tectona grandis trees.

Laasasenaho et al. (2005) estudando a espécie Picea abies também verificaram a mesma tendência do afilamento da casca ao modelar por meio de funções de taper, a espessura da casca em função da altura relativa das árvores. Optou-se pela relativização das variáveis visto a necessidade de padronização da informação uma vez que há um número distinto de amostras em árvores de diferentes alturas.

Já para a relação entre a EC e o di foi observado um padrão linear crescente, semelhante ao observado por Cordero e Kanninen (2003) para a mesma espécie. Isso explica a intensiva utilização do di como variável independente para modelar a relação entre o diâmetro com e sem casca a qualquer altura do fuste (CORDERO; KANNINEN, 2003; LI; WEISKITTEL, 2011; STÄNGLE et al., 2015).

Os coeficientes estimados e as estatísticas $r_{y \hat{y}}$ e RQME\% obtidas no ajuste dos modelos para predizer espessura de casca são apresentados na Tabela 2. Devido a não significância da variável associada ao parâmetro " $\beta 5$ " para o modelo polinomial este não foi utilizado no processo de modelagem.

A relação funcional pelo modelo I $[E C=f(h r e l)]$ explicou apenas $56 \%\left(\mathrm{R}^{2}\right)$ da variação da espessura da casca ao longo fuste, além do erro médio apresentar-se elevado (>26\%). A baixa qualidade das estimativas está associada à ampla variação de idade das árvores amostradas ( 6 a 34 anos), já que a medida que a idade aumenta a espessura da casca é maior (MOLINA et al., 2016). Já pelo modelo II [EC=f(di)] houve uma melhora expressiva, apresentando uma explicação de até $72 \%\left(\mathrm{R}^{2}\right)$ e consequentemente uma redução do erro médio. Essa melhora é atribuída devido a variável independente di captar parte da variação da idade das árvores, dada a correlação existente entre essas variáveis.

Visando melhorar a acurácia do modelo e reduzir mais o erro da estimativa, um novo ajuste foi efetuado inserindo a idade como efeito aleatório, buscando explicar mais da variação dos dados referentes a espessura da casca ao longo do tronco das árvores de teca. Na modelagem de efeitos

Tabela 2. Coeficientes e estatísticas dos modelos utilizados para estimativa da espessura da casca de Tectona grandis. Table 2. Coefficients and statistics of the models used to estimate the thickness of the bark of Tectona grandis.

\begin{tabular}{|c|c|c|c|c|}
\hline Parâmetro & Estimativa & $\operatorname{Pr}(>|t|)$ & $r_{y \hat{y}}$ & RQEM \% \\
\hline \multicolumn{5}{|c|}{ Modelo polinomial } \\
\hline$\hat{\beta}_{0}$ & 0,79306 & $<2.10^{-16}$ & \multirow{5}{*}{0,749} & \multirow{5}{*}{26,869} \\
\hline$\hat{\beta}_{1}$ & $-1,91255$ & $6.10^{-12}$ & & \\
\hline$\hat{\beta}_{2}$ & 4,47327 & $7.10^{-3}$ & & \\
\hline$\hat{\beta}_{3}$ & $-5,47885$ & $1,0.10^{-02}$ & & \\
\hline$\hat{\beta}_{4}$ & 2,38690 & $4,1.10^{-02}$ & & \\
\hline \multicolumn{5}{|c|}{ Modelo linear simples } \\
\hline$\hat{\beta}_{0}$ & 0,18663 & $<2.10^{-16}$ & \multirow{2}{*}{0,849} & \multirow{2}{*}{21,457} \\
\hline$\hat{\beta}_{I}$ & 0,02015 & $<2.10^{-16}$ & & \\
\hline
\end{tabular}

$\hat{\beta}_{i}=$ coeficientes de regressão estimados; $r_{y \hat{y}}=$ coeficiente de correlação; RQEM\% = erro padrão relativo. 
Vendruscolo et al. - Modelagem da espessura e percentual de casca em

árvores de Tectona grandis L.f

mistos, a variável aleatória foi inserida decompondo apenas o parâmetro " $\hat{\beta}_{0}$ ", haja visto que a decomposição dos outros resultou em não significância dos parâmetros estimados.

Observou-se que os modelos com efeitos mistos foram superiores em relação à modelagem de efeito fixo, tomado como base as estatísticas de ajuste ( $r_{y \hat{y}}$ e RQEM\%). Ao avaliar a relação funcional $E C=f(h r e l)$, verificou-se que o valor do erro padrão relativo foi reduzido de $26,9 \%$ para $17,9 \%$, e para o coeficiente de correlação foi verificado uma superioridade na explicação pelas variáveis independentes

Tabela 3. Coeficientes e estatísticas de ajuste dos modelos de efeito misto utilizados para estimativa da espessura da casca de Tectona grandis.

Table 3. Coefficients and adjustment statistics of the mixed effect models used to estimate the thickness of Tectona grandis bark.

\begin{tabular}{|c|c|c|c|c|c|c|c|}
\hline \multirow[t]{2}{*}{ Id. } & $\hat{\boldsymbol{\beta}}_{0}$ & $\hat{\boldsymbol{\beta}}_{1}$ & $\hat{\boldsymbol{\beta}}_{2}$ & $\hat{\boldsymbol{\beta}}_{3}$ & $\hat{\boldsymbol{\beta}}_{4}$ & \multirow{2}{*}{$r_{y \hat{y}}$} & \multirow{2}{*}{ RQEM \% } \\
\hline & aleatório & fixo & fixo & fixo & Fixo & & \\
\hline \multicolumn{8}{|c|}{ Modelo polinomial } \\
\hline 6 & 0,68555 & \multirow{6}{*}{$-1,89788$} & & \multirow{6}{*}{$-5,84948$} & \multirow{6}{*}{2,56500} & \multirow{6}{*}{0,898} & \multirow{6}{*}{17,858} \\
\hline 7 & 0,69498 & & & & & & \\
\hline 10 & 0,79360 & & & & & & \\
\hline 16 & 0,84448 & & 4,62435 & & & & \\
\hline 17 & 0,81322 & & & & & & \\
\hline 34 & 0,97903 & & & & & & \\
\hline \multicolumn{8}{|c|}{ Modelo linear simples } \\
\hline 6 & 0,20032 & \multirow{6}{*}{0,02135} & - & - & - & \multirow{6}{*}{0,862} & \multirow{6}{*}{20,557} \\
\hline 7 & 0,15486 & & - & - & - & & \\
\hline 10 & 0,22757 & & - & - & - & & \\
\hline 16 & 0,13888 & & - & - & - & & \\
\hline 17 & 0,18493 & & - & - & - & & \\
\hline 34 & 0,13895 & & - & - & - & & \\
\hline
\end{tabular}

Id. = idade (anos); $\hat{\beta}_{i}=$ coeficientes de regressão estimados; $r_{y \hat{y}}=$ coeficiente de correlação; RQEM\% = erro padrão relativo.

de aproximadamente 16,6\% (Tabela 3). Enquanto para o modelo II, o ganho em precisão foi de apenas $1,5 \%$ para o $r_{y \hat{y}}$ e $0,9 \%$ para o RQEM.

As análises gráficas (Figuras 4 e 5) confirmam a superioridade do modelo com efeito misto que utiliza a altura relativa como variável independente, e pouca melhora quando se utiliza o diâmetro. Houve uma redução na amplitude da dispersão dos resíduos, indicando maior acurácia. Essa melhoria
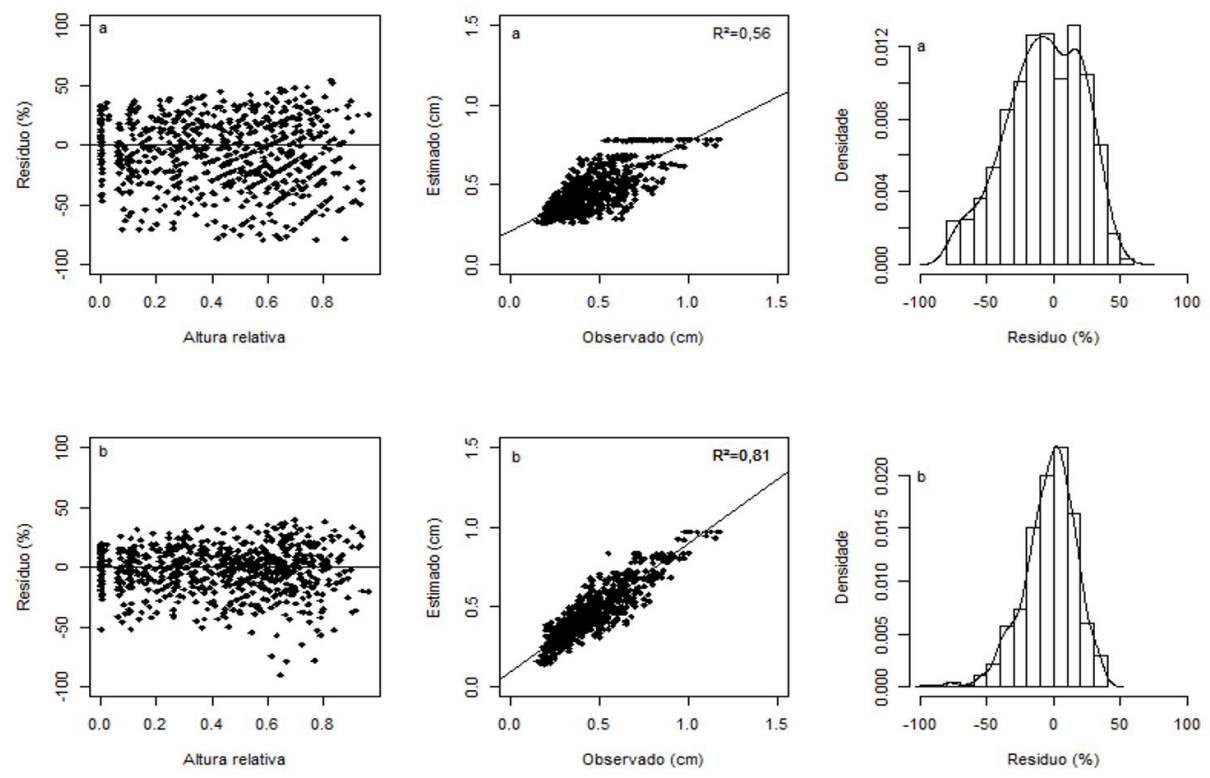

Figura 4. Resíduos, valores observados e estimados e histogramas dos resíduos do modelo polinomial fixo (a) e com efeito misto (b).

Figure 4. Residues, observed and estimated values and histograms of the residues of the fixed polynomial model (a) and with mixed effect (b). 

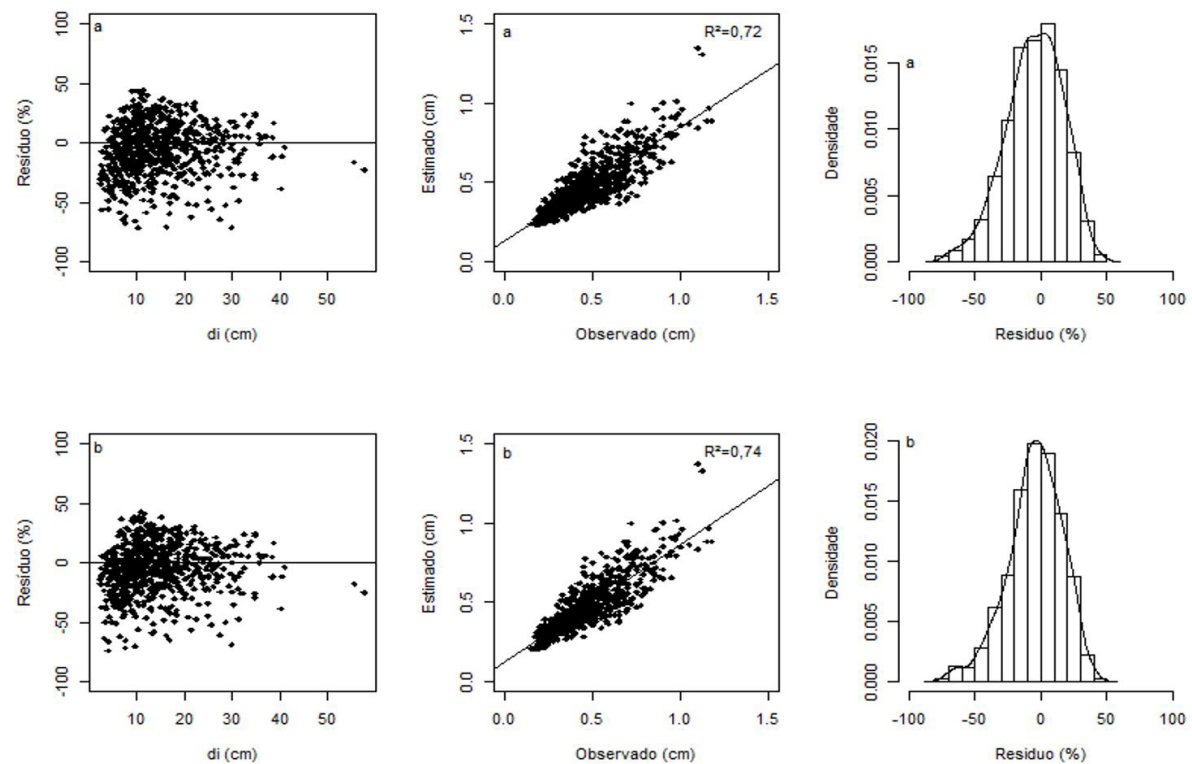

Figura 5. Resíduos, valores observados e estimados e histogramas dos resíduos do modelo II fixo (a) e com efeito misto (b).

Figure 5. Residues, observed and estimated values and histograms of model II fixed residues (a) and with mixed effect (b)

é mais evidente quando analisado os valores estimados versus os observados, em que os pontos estão mais concentrados da reta $(0,1)$.

Pode-se inferir que o modelo polinomial com efeito misto foi mais eficiente nas estimativas da espessura de casca. Verificou-se que a declividade da linha de tendência entre os valores observados e estimados, apresentam uma menor dispersão dos dados e o intercepto $(\beta 0)$ próximo a 0 e inclinação ( $\beta 1$ ) próxima a 1, o que é desejável e significa que os valores são coincidentes segundo Campos e Leite (2013). Ainda, ao comparar os dois modelos empregados, deve-se considerar que o modelo I é mais vantajoso do ponto de vista operacional, pois dispensa a medição de diâmetros em diferentes alturas do fuste como é o caso do modelo II.

De forma complementar, os resíduos em forma de histogramas também mostram melhoras acentuadas do modelo de efeito misto. Resultados semelhantes foram obtidos por Li e Weiskittel (2011), em que os autores destacaram que o uso de modelos mistos para estimar a espessura de casca proporciona um aumento de precisão nas estimativas.

Mesmo que verificado uma ampla variação dos dados entorno da linha estimada, o efeito aleatório da idade proporciona melhoraria expressiva nas estimativas (Figura 6). Isso ocorre porque a variável idade influência de forma significativa a espessura da casca das árvores, como também observado por Laasasenaho et al. (2005) para Picea abies e Molina et al. (2016) para Araucaria araucana. Além da variável idade, outras como a variação geoespacial, produtividade do site e diferentes taxas de crescimento individual podem influenciar a espessura da casca (STÄNGLE; SAUTER; DORMANN et al., 2017). Ainda, estudos feitos por Stängle et al. (2015) para Picea abies, revelaram que a variação da espessura da casca pode variar dentro de uma única árvore.

Como comprovado neste trabalho, a espessura da casca vai diminuindo expressivamente no sentido da base até o topo, situação semelhante a conicidade da árvore, que é o decréscimo de diâmetro ao longo do fuste (Figura 6). Nas maiores alturas a casca é mais delgada por ser mais jovem, enquanto na base, a proporção é maior devido certa predominância de casca morta externa, que segundo Paine et al. (2010) é um mecanismo que a planta tende a manter para se defender de adversidades que podem afetar seu câmbio e xilema. Cordero e Kanninem (2003) observaram em árvores de teca entre 5 e 47 anos na Costa Rica, que o volume da casca aumentam linearmente com a idade, devido ao aumento da espessura e das dimensões das árvores.

As equações ajustadas para estimativa da porcentagem de casca apresentaram estatísticas de coeficiente de correlação, com valores superiores a 0,80, e erro médio variando de 13 a 13,8\% (Tabela 4). O modelo biexponencial apresentou vantagem em relação ao exponencial simples conforme os indicadores estatísticos. 
Vendruscolo et al. - Modelagem da espessura e percentual de casca em árvores de Tectona grandis L.f

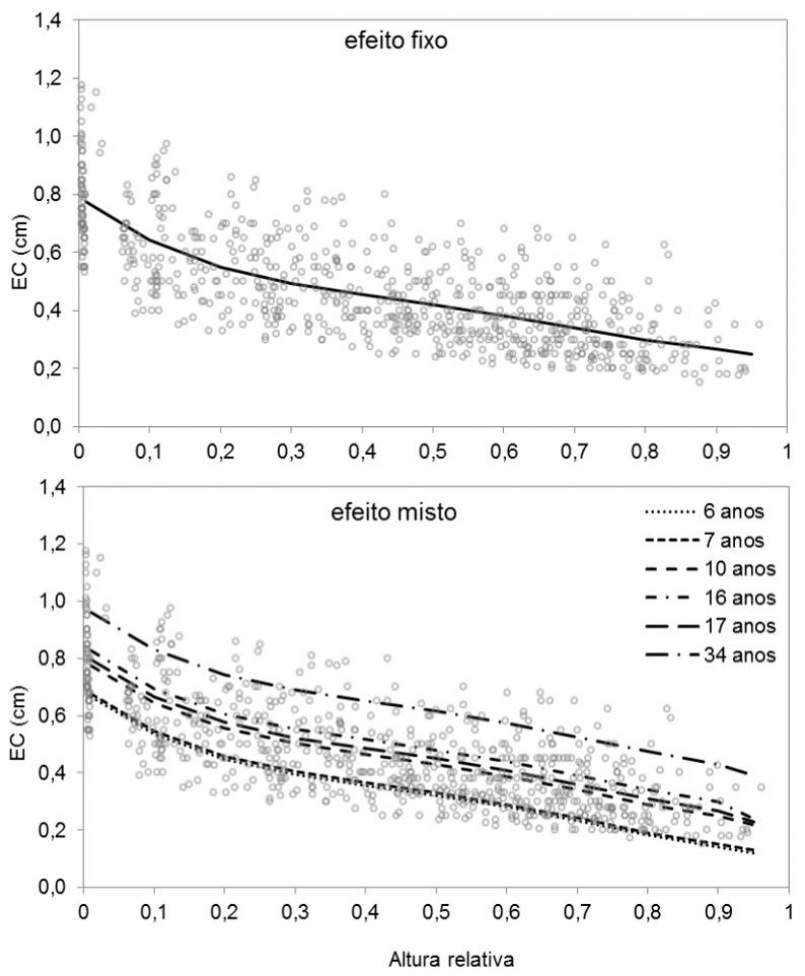

Figura 6. Curvas de afilamento da casca de Tectona grandis por modelagem de efeito fixo e misto (modelo I). Figure 6. Taper curves of Tectona grandis bark by fixed and mixed effect modeling (model I).

Tabela 4. Coeficientes e estatísticas de ajuste para as equações de porcentagem de casca de Tectona grandis. Table 4. Coefficients and adjustment statistics for the equations of bark of Tectona grandis.

\begin{tabular}{|c|c|c|c|c|}
\hline Parâmetro & Estimativa & $\operatorname{Pr}(>|t|)$ & $r_{y \hat{y}}$ & RQEM \% \\
\hline \multicolumn{5}{|c|}{ Modelo exponencial } \\
\hline$\beta_{0}$ & 20,92958 & $<2.10^{-16}$ & \multirow[b]{2}{*}{0,814} & \multirow[b]{2}{*}{13,747} \\
\hline$\beta_{1}$ & $-3,80318$ & & & \\
\hline \multicolumn{5}{|c|}{ Modelo biexponencial } \\
\hline$\beta_{0}$ & 22,54860 & $3,2.10^{-02}$ & \multirow{4}{*}{0,835} & \multirow{4}{*}{13,015} \\
\hline$\beta_{1}$ & $-1,86440$ & $3,9.10^{-02}$ & & \\
\hline$\beta_{2}$ & 14,4770 & $1,7.10^{-02}$ & & \\
\hline$\beta_{3}$ & $-4,5393$ & $1,7.10^{-05}$ & & \\
\hline
\end{tabular}

$\hat{\beta}_{i}=$ coeficientes de regressão estimados; $r_{y \hat{y}}=$ coeficiente de correlação; RQEM\% = erro padrão relativo.

Pode-se observar que no melhor ajuste, o diâmetro explica apenas cerca de $70 \%$ da porcentagem de casca nas árvores de teca. Resultados semelhantes foram obtidos por Tewari e Mariswamy (2013) onde reportaram que essa modelagem [\%casca $f(D A P)]$, pode apresentar ajustes com menor grau de explicação entre a variável independente com a dependente.

Ao analisar as estimativas das duas equações por meio dos gráficos de resíduos, valores observados versus estimados e os histogramas de densidade residual, observou-se que a equação proveniente do ajuste do modelo biexponencial foi mais acurada (Figura 7).

Embora sejam poucas as investigações sobre a modelagem do porcentual de casca em povoamentos de teca, estudos realizados por Cordero e Kanninen (2003), Leite et al. (2011) e Tewari e Mariswamy (2013), apontam a mesma tendência de decréscimo da média do percentual de casca com o aumento do DAP, conforme também observado na Figura 8.

Essa redução do percentual de casca é esperada, visto que à medida que aumenta o DAP, há um incremento volumétrico exponencial, e consequentemente uma menor proporção de casca para os 

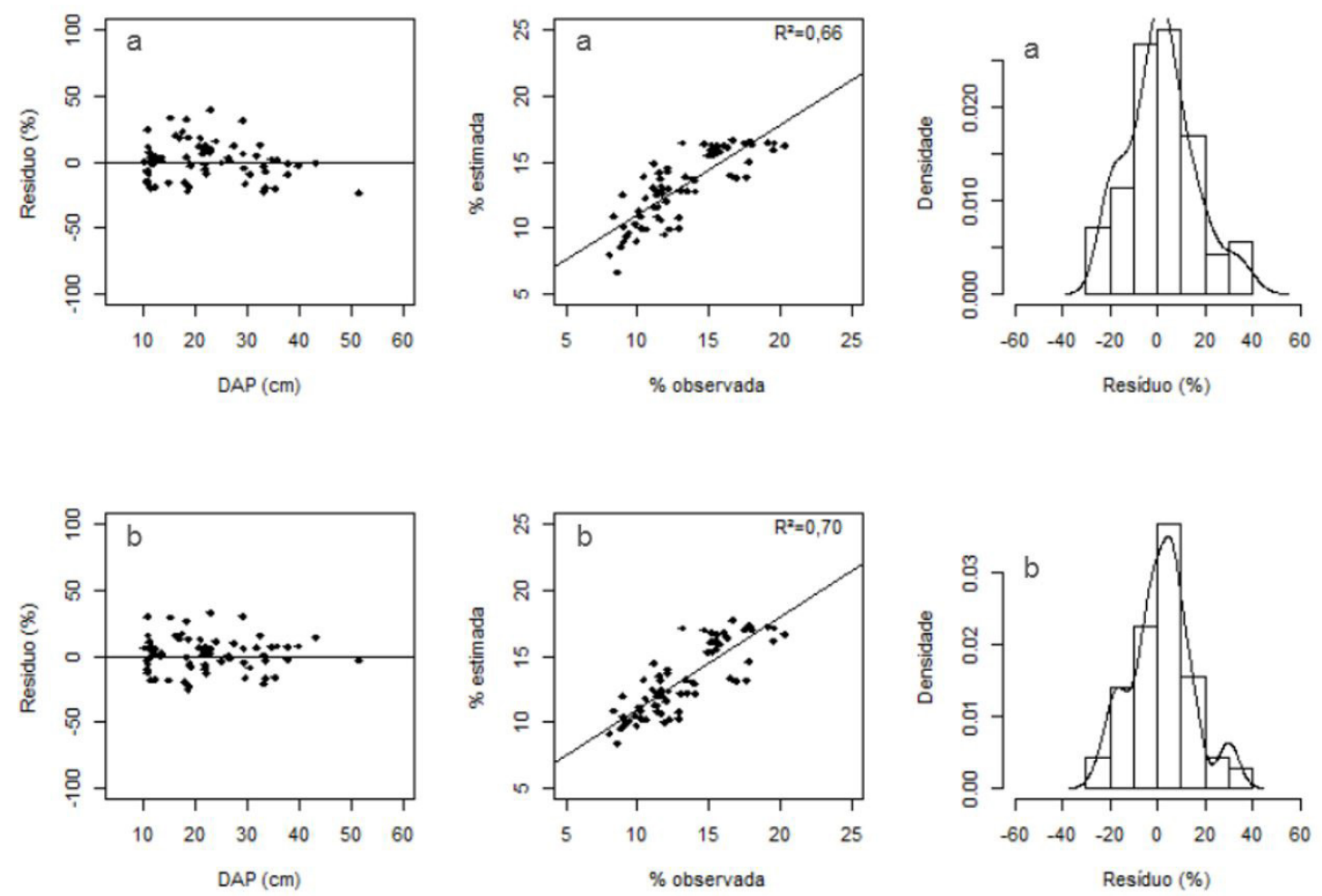

Figura 7. Resíduos, valores observados e estimados e histogramas dos resíduos dos modelos exponencial (a) e biexponencial (b) para estimativas de porcentagem de casca de Tectona grandis.

Figure 7. Residues, observed versus estimated values and histograms of residues for the exponential (a) and bi-exponential (b) models for percentage estimates of bark of Tectona grandis.

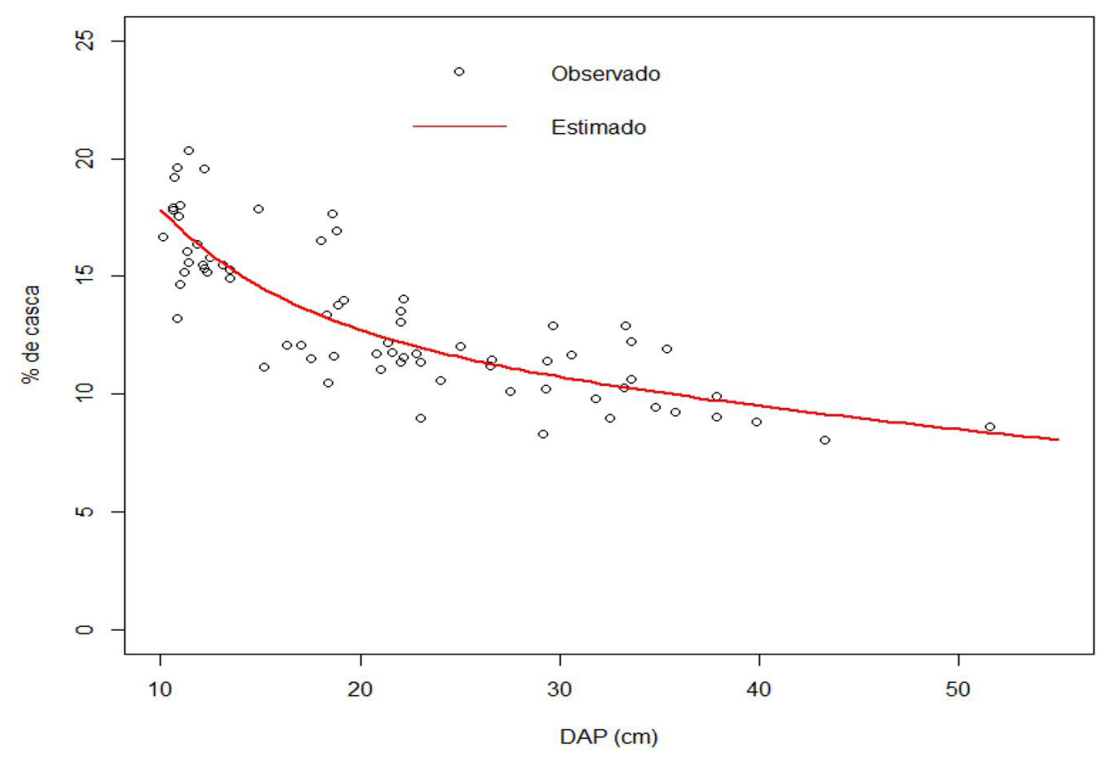

Figura 8. Curva do percentual de casca em função do DAP de Tectona grandis estimada pelo modelo biexponencial. Figure 8. Percentage curve of bark related to $\mathrm{DBH}$ of Tectona grandis estimated by the bi-exponential model.

indivíduos maiores (FIGUEIREDO et al., 2005; FINGER, 2006). Além de que essa tendência é mais evidente quando relacionada com o DAP do que com a idade, segundo Cordero e Kanninen (2003).

Deve-se levar em consideração que os percentuais de casca apresentados neste trabalho se referem às medições de casca "seca", obtida dos discos utilizados na análise de tronco, que devido à desidratação é comum ocorrer uma retração expressiva na espessura da casca de espécies florestais (FRITTZ, 1958). Sendo assim, se considerado as medições de casca "verde", esses percentuais certamente serão superiores, podendo representar valores altamente significativos em relação ao volume total das árvores, principalmente em povoamentos jovens devido as árvores apresentarem menores diâmetros. 
Vendruscolo et al. - Modelagem da espessura e percentual de casca em

árvores de Tectona grandis L.f

Nesse contexto, estudos complementares devem ser feitos com objetivo de avaliar essa retração para a espécie em questão.

\section{CONCLUSÃO}

O modelo polinomial com a variável independente altura relativa e a idade como efeito aleatório mostrou-se eficiente para a predição da espessura da casca ao longo do fuste de árvores de Tectona grandis. Ainda, a utilização desse modelo permite uma vantagem operacional em relação ao modelo I, pois dispensa a medição de diâmetros ao longo do fuste das árvores.

O conteúdo percentual de casca em árvores em pé de Tectona grandis pode ser estimado com precisão, utilizando um modelo biexponencial com apenas o DAP como variável regressora.

\section{REFERÊNCIAS BIBLIOGRÁFICAS}

ALVARES, C. A.; STAPE, J. L.; SENTELHAS, P. C.; GONÇALVES, J. L. M.; SPAROVEK, G. Köppen's climate classification map for Brazil. Meteorologische Zeitschrift, v. 22, n. 6, p. 711-728, 2013.

BROOKS, J. R.; JIANG, L. Comparison of prediction equations for estimating inside bark diameters for yellowpoplar, red maple, and red pine in West Virginia. Northern Journal of Applied Forestry, v. 26, n. 1, p. 5-8, 2009.

CAMPOS, J. C. C.; LEITE, H. G. Mensuração florestal: perguntas e respostas. 4. ed. Viçosa: UFV, 2013. 605 p.

CHAVES, A. G. S.; DRESCHER, R.; CALDEIRA, S. F.; MARTINEZ, D. T.; VENDRUSCOLO, D. G. S. Capacidade produtiva de Tectona grandis L.f no Sudoeste de Mato Grosso. Scientia Forestalis, v. 44, n. 104, p. 415- 424, 2016.

CORDERO, L.; KANNINEN, M. Heartwood, sapwood and bark content, and wood dry density of young and mature teak (Tectona grandis) trees grown in Costa Rica. Silva Fennica, v. 37, n. 1, p. 45-54, 2003.

DORUSKA, P. F.; PATTERSON, D.; HARTLEY, J.; HURD, M.; HART, T. Newer technologies and bioenergy bring focus back to bark factor equations. Journal of Forestry, v. 107, n.1, p. 38-43, 2009.

DRESCHER, R.; NUNES, G. M.; MARTINEZ, D. T.; PELISSARI, A. L Capacidade produtiva do sítio em povoamentos jovens de Tectona grandis L. f. de duas regiões do estado de Mato Grosso - Brasil. Revista Brasileira de Biometria, v. 34, n. 2, p. 233-242, 2016.

FAO - FOOD AND AGRICULTURE ORGANIZATION OF THE UNITED NATIONS. Global teak trade in the aftermath of Myanmar's log export ban. 20015 (Planted Forests and Trees Working Paper FP/49/E). Disponível em: < http://www.fao.org/3/a-i5023e.pdf >. Acesso em 10 ago 2017.

JANG, W.; KEYES, C. R.; GRAHAM, J. M. Evaluation of predictive models for Douglas-fir bark thickness at breast height following 12 biomass harvest treatments. Biomass and Bioenergy, v. 84, p. 118 - 123, 2016.

FIGUEIREDO, O. B.; SCOLFORO, J. R. S.; OLIVEIRA, A.D. Estimativa do percentual de casca e fator de forma em povoamentos jovens de teca (Tectona grandis L.f). Rio Branco: Embrapa Acre, 2005. 5 p. (Embrapa Acre. Documentos, 165).

FINGER, C. A. Biometria Florestal. Santa Maria. Universidade Federal de Santa Maria, 2006. 248 p.

FRITTZ, H. C. An analysis of radial growth of beech in a Central Ohio Forest during 1954 - 55. Ecology, v. 39, n. 4, p. 705-720, 1958.

GAVA, F. H. Danos na copa: crescimento, produção e desbaste em povoamentos clonais e seminais de Teca em duas regiões de Mato Grosso. 2015. 77 p. Dissertação (Mestrado em Ciências Florestais e Ambientais) Universidade Federal de Mato Grosso, Cuiabá, 2015.

HARKIN, J. M.; ROWE, J. W. Bark and its possible uses. Madison: USDA Forest Service, 1971. 56 p. (Forest Products Laboratory Report FLP-091).

KITIKIDOU, K.; PAPAGEORGIOU, A.; MILEOS, E.; STAMPOULIDIS, A. A bark thickness model for Pinus halepensis in Kassandra, Chalkidiki (Northern Greece). Silva Balcanica, v. 15, n. 1, p. 47-55, 2014. 
LAASASENAHO, J.; MELKAS, T.; ALDEN, S. Modelling bark thickness of Picea abies with taper curves. Forest Ecology and Management, v. 206, n. 1, p. 35-47, 2005.

LEITE, H. G.; OLIVEIRA NETO, R. R. D.; MONTE, M. A.; FARDIN, L.; ALCANTARA, A. M. D.; BINOTI, M. L. M. S.; CASTRO, R. V. O. Modelo de afilamento de cerne de Tectona grandis Lf. Scientia Forestalis, v. 39, n. 89, p. 053-059, 2011.

LI, R.; WEISKITTEL, A. R. Estimating and predicting bark thickness for seven conifer species in the Acadian Region of North America using a mixed-effects modeling approach: comparison of model forms and subsampling strategies. European Journal of Forest Research, v. 130, n. 2, p. 219-233, 2011.

MOLINA, J. G. A.; HADAD, M. A.; DOMÍNGUEZ, D. P.; ROIG, F. A. Tree age and bark thickness as traits linked to frost ring probability on Araucaria araucana trees in northern Patagonia. Dendrochronologia, v. 37, p. 116-125, 2016.

MOYA, R.; BOND, B.; QUESADA-PINEDA, H. A review of heartwood properties of Tectona grandis trees from fast-growth plantations. Wood Science and Technology, v. 48, n. 2, p. 411-433, 2014.

PAINE, C. E. T.; STAHL, C.; COURTOIS, E. A.; PATIÑO, S.; SARMIENTO, C.; BARALOTO, C. Functional explanations for variation in bark thickness in tropical rain forest trees. Functional Ecology, v. 24, n. 6, p. $1202-1210,2010$.

PINHEIRO, J. C.; BATES, D. M. Mixed-effects models in S and S-PLUS. New York: Springer-Verlag, 2000.528 p.

R CORE TEAM. R: A language and environment for statistical computing. Vienna: R Foundation for Statistical Computing, 2017.

STÄNGLE, S. M.; SAUTER, U. H.; DORMANN, C. F. Comparison of models for estimating bark thickness of Picea abies in southwest Germany: the role of tree, stand, and environmental factors. Annals of Forest Science, v. 74, n.16, p.1-10, 2017.

STÄNGLE, S. M.; WEISKITTEL, A. R.; DORMANN, C. F.; BRÜCHERT, F. Measurement and prediction of bark thickness in Picea abies: assessment of accuracy, precision, and sample size requirements. Canadian Journal of Forest Research, v. 46, n. 1, p. 39-47, 2015.

TEWARI, V. P.; MARISWAMY, K. M. Heartwood, sapwood and bark content of teak trees grown in Karnataka, India. Journal of forestry research, v. 24, n. 4, p. 721-725, 2013.

VENDRUSCOLO, D.; G.; S. Modelagem aplicada ao manejo de povoamentos de Tectona grandis L.f em Mato Grosso. 2017. 87 p. Dissertação (Mestrado em Ciências Florestais e Ambientais) - Universidade Federal de Mato Grosso, Cuiabá, 2017.

Recebido em: 15/11/2017

Aceito em: 08/08/2018

Sci. For., Piracicaba, v. 47, n. 121, p. 139-149, mar. 2019

DOI: dx.doi.org/10.18671/scifor.v47n121.14 